\title{
P 018 TRANSFORMING PALLIATIVE AND END OF LIFE CARE SERVICES THROUGH A WHOLE SYSTEMS APPROACH
}

B O'Hara, N Davidson, C McClements, D Wilson. Transforming Your Palliative and End of Life Care Programme, Marie Curie

\subsection{6/bmjspcare-2014-000838.21}

Introduction Current strategies to address palliative and end of life care in Northern Ireland emphasise the importance of providing care as close to home as possible. In order to achieve this the transforming your palliative and end of life care programme in Northern Ireland has adopted a whole systems approach to service redesign which promotes meaningful patient, family and carer involvement.

Aim(s) and method(s) Through a whole systems approach enable stakeholders to make suggestions as to how services could be improved, changed or developed; and generate new ideas for providing care for people towards the end of life at home or as close to home as possible.

Key stakeholders were identified across statutory, voluntary/community and independent sectors including patients, families and carers. Seven workshops 


\section{Abstracts}

were held across Northern Ireland. Participant booklets were used to capture all suggested ideas. Meetings also took place with service users and carers individually and in small groups. Recorded ideas were systematically themed by the programme team before undergoing a two-stage shortlisting process using set criteria.

Results 412 stakeholders attended the workshops. 648 ideas were recorded resulting in and informing eight priority initiatives being taken forward: identification and planning; enhanced access to services at home; day hospice; support for patients and carers; pharmacy; training for healthcare providers; ambulance; discharge and equipment.

Conclusion(s) Through a whole systems approach stakeholders across various sectors were able to engage in meaningful discussion to transform how palliative care will be delivered in Northern Ireland. 